\section{La fracture de la hanche et la thérapie par inhibiteurs de la pompe à protons : Un document de principes}

Paul Moayyedi, B. Sc. MB CLB Ph. D. MPH FRCP (Londres) FRCPC Comité des affaires cliniques de l'ACG

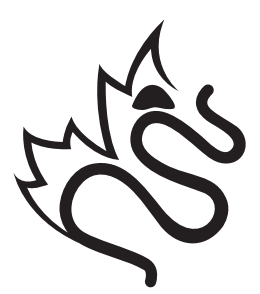

L'Association Canadienne de Gastroentérologie

For English see page 855
L es médias se sont récemment intéressés à la possibilité que la Lescription d'inhibiteurs de la pompe à protons (IPP) soit reliée à un risque accru de fracture de la hanche, selon des données tirées de bases de données du Royaume-Uni (RU) (1) et du Canada (2). À la lumière de ces données, l'Association canadienne de gastroentérologie a préparé le document de principes suivant sur l'association entre la thérapie par IPP et la fracture de la hanche. Ce document de principes est détaillé ailleurs (3).

Yang et coll. (1) ont rendu compte d'une étude cas-témoin à plusieurs critères de classification au moyen de la base de données de recherche en pratique générale du RU, dans laquelle on avait sélectionné des patients de plus de 50 ans ayant subi un incident de fracture de la hanche et des témoins appariés selon l'âge et le sexe. Il y avait 13556 cas de fracture de la hanche et 135386 témoins. La thérapie par IPP pendant plus d'un an s'associait à une augmentation du risque de fracture de la hanche (RRR 1,44; $95 \%$ IC 1,30 à 1,59). Targownik et coll. (2) ont rendu compte d'une autre étude cas-témoin à plusieurs critères de classification au moyen du Manitoba Population Health Research Data Repository, dans laquelle 15792 autres patients ayant subi une fracture reliée à l'ostéoporose étaient comparés à 47289 témoins. Ils n'ont relevé aucune association globale entre l'utilisation des IPP et les fractures avant une exposition d'au moins cinq ans, lorsque le risque de fracture ostéoporotique devenait significatif (RRR 1,62; 95 \% IC 1,02 à 2,58). De même, le risque de fracture de la hanche devenait important après une exposition aux IPP pendant sept ans (RRR 4,55; 95 \% IC 1,68 à 12,29).

La signification clinique de ces données est incertaine, parce que d'après l'extrapolation de ces nombres (en supposant que 1,8 patient sur 1000 a une fracture de la hanche [1]), 1263 patients devaient prendre des IPP pendant plus d'un an pour subir une fracture de la hanche, mais d'après l'étude canadienne, le nombre nécessaire pour nuire diminuerait considérablement au bout de sept ans.

Ces études font appel à des bases de données fiables, sont tenues avec rigueur et bien analysées. L'exposé contenu dans ces deux articles insiste pour postuler une association causale. Les grandes bases de données fournissent une excellente occasion d'évaluer les bienfaits et les dommages des interventions médicales, mais il faut faire preuve de prudence avant de postuler que la dernière constatation représente un risque pour les patients. Étant donné l'accès facile aux bases de données, il est possible de vérifier des millions d'associations, mais au sein d'une même hypothèse, il existe divers modes de partage des données (p. ex., durée du traitement, dose du traitement, durée du suivi, âge du patient, homme ou femme). Les chercheurs et les revues scientifiques ont tendance à faire ressortir les observations positives et à minimiser le rôle du hasard ou des variables confusionnelles. Ces aspects sont moins susceptibles d'avoir de l'importance lorsqu'on découvre une association uniforme. Un éditorial (4) qui accompagnait l'étude canadienne citait également une troisième étude de base de donné danoise étayant une association entre la thérapie par IPP et la fracture (5). Il est intéressant de constater que l'étude danoise comptait un nombre beaucoup plus important de cas (124 665) que les deux autres études, mais que ses conclusions étaient beaucoup plus tempérées et qu'elle était publiée dans une revue de moindre importance.

Les conclusions de l'étude danoise étaient circonspectes à cause d'un manque de cohérence dans les données et de l'absence d'information sur la réaction aux doses. Par exemple, le risque de prendre moins de 25 doses d'IPP en un an semblait plus élevé que celui (toujours statistiquement significatif) de prendre une dose par jour pendant la même période. Aucune hypothèse plausible d'un point de vue biologique ne semble expliquer pourquoi une utilisation si peu fréquente de la thérapie par IPP accroîtrait le risque de fracture. Il y avait aussi un manque de cohérence dans les études du RU et du Canada. L'étude du RU fait état d'un risque accru de fracture de la hanche après une thérapie par IPP d'un an. Le risque semblait peu augmenter au cours des quatre années suivantes, tandis que l'étude canadienne décelait une association significative au bout de seulement sept ans. Targownik et coll. (2) ont avancé que la base de données du RU incluait peut-être des patients qui prenaient des IPP depuis beaucoup plus longtemps, mais il ne s'agit là que de spéculations.

De plus, une étude de cohortes canadienne (6) a fait un suivi auprès de patients âgés de l'Ontario qui prenaient de la warfarine ( $n=52$ 701), une hormonothérapie thyroïdienne substitutive $(n=40555)$, des corticoïdes oraux $(n=43915)$ et une thérapie par IPP ( $n=60383$ ) pendant cinq ans. Les personnes qui prenaient de la warfarine ne couraient pas un risque plus élevé de fracture de la

L'ACG est fière de présenter ses bienfaiteurs :

Abbott Canada

Pentax Canada Inc
AstraZeneca Canada Inc

Procter \& Gamble Pharmaceuticals
Axcan Pharma Inc

Schering-Plough Canada Inc
Olympus Canada Inc

UCB Pharma Inc 
plus élevé que celui de la thérapie par IPP (RRR 1,44; 95 \% IC $1,21$ à 1,70$)$. Il se peut que la warfarine s'associe également à un risque accru de fracture de la hanche, mais Targownik et coll. (2) ont décelé peu d'augmentation du risque de fracture chez les personnes qui prenaient des anticoagulants $(14,5 \%$ des cas de fracture chez les personnes qui prenaient des anticoagulants par rapport à 13, 4 \% des cas témoins).

Enfin, il faut tenir compte de la plausibilité biologique. Il est proposé que la suppression acide pourrait réduire l'absorption du calcium et accroître le risque de fracture (1). Toutefois, le rôle du pH dans l'absorption du calcium demeure controversé. La dissolution du carbonate de calcium dépend du $\mathrm{pH}$ in vitro, mais sa signification clinique est incertaine puisque le calcium est absorbé par l'intestin grêle, où le $\mathrm{pH}$ n'est pas acide, quel que soit le $\mathrm{pH}$ de l'estomac. Une analyse bibliographique (7) a permis de repérer sept essais aléatoires qui évaluaient l'absorption du calcium avec suppression acide chez des sujets en santé ou atteints d'une maladie ulcéreuse. La plupart des études comportaient d'importants problèmes méthodologiques, et elles portaient toutes sur de petits échantillons. Quatre études n'ont constaté aucune répercussion sur l'absorption de calcium, tandis que trois ont constaté une diminution de l'absorption. Il faudra d'autres travaux pour rendre l'association entre la fracture et la thérapie par IPP plausible d'un point de vue biologique. De plus, d'après certaines études, la

\section{RÉFÉRENCES}

1. Yang YX, Lewis JD, Epstein S, Metz DC. Long-term proton pump inhibitor therapy and risk of hip fracture. JAMA 2006;296:2947-53.

2. Targownik LE, Lix LM, Metge CJ, Prior HJ, Leung S, Leslie WD. Use of proton pump inhibitors and risk of osteoporosis-related fractures. CMAJ 2008; 179: 319-26.

3. Moayyedi P, Cranney A. Hip fracture and proton pump inhibitor therapy: Balancing the evidence for benefit and harm.

Am J Gastroenterol 2008;in press

4. Richards JB, Goltzman D. Proton pump inhibitors: Balancing the benefits and potential fracture risks. CMAJ 2008;179:306-7. thérapie par IPP peut inhiber la résorption osseuse (8), ce qui peut être protecteur contre le risque de fracture.

Les IPP sont l'une des catégories de médicaments les mieux tolérées sur le marché. Ils ont amélioré la qualité de vie d'innombrables patients atteints d'une maladie reliée à l'acide. Il y a des risques à prescrire des médicaments, et tous les cliniciens prescripteurs devraient en tenir compte. La thérapie par IPP ne devrait être prescrite que pour des indications à l'égard desquelles les bienfaits sont démontrés ou probables, et il faudrait en réévaluer régulièrement la nécessité. Ce principe s'applique particulièrement aux personnes âgées de santé fragile présentant de multiples comorbidités et qui prennent plusieurs médicaments différents, ce qui accrôit la possibilité d'interactions entre médicaments. Ce message s'applique à tous les médicaments prescrits par les cliniciens, y compris la thérapie par IPP. Les données actuelles n'étayent pas des précautions particulières avant de prescrire une thérapie par IPP en raison des préoccupations relatives aux fractures de la hanche. Aucune donnée probante convaincante ne peut démontrer une association causale, mais on ne pourra jamais en exclure la possibilité. L'Association canadienne de gastroentérologie invite les professionnels de la santé à toujours se tenir au courant des publications médicales sur le sujet et diffusera d'autres conseils si plus d'information au sujet des dommages possibles de la thérapie par IPP devient disponible.

5. Vestergaard P, Rejnmark L, Mosekilde L. Proton pump inhibitors: Histamine $\mathrm{H} 2$-receptor antagonists, and other antacid medications and the risk of fracture. Calcif Tissue Int 2006;79:76-83.

6. Mamdani M, Upshur RE, Anderson G, Bartle BR, Laupacis A. Warfarin therapy and risk of hip fracture among elderly patients. Pharmacotherapy 2003;23:1-4.

7. Wright MJ, Proctor DD, Insogna KL, Kerstetter JE. Proton pumpinhibiting drugs, calcium homeostasis, and bone health. Nutr Rev 2008;66:103-8.

8. Tuukkanen J, Vaananen HK. Omeprazole: A specific inhibitor of $\mathrm{H}^{+}-\mathrm{K}^{+}$-ATPase, inhibits bone resorption in vitro. Calcif Tissue Int 1986;38:123-5. 


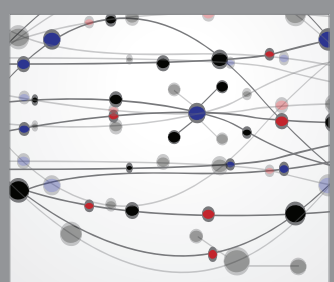

The Scientific World Journal
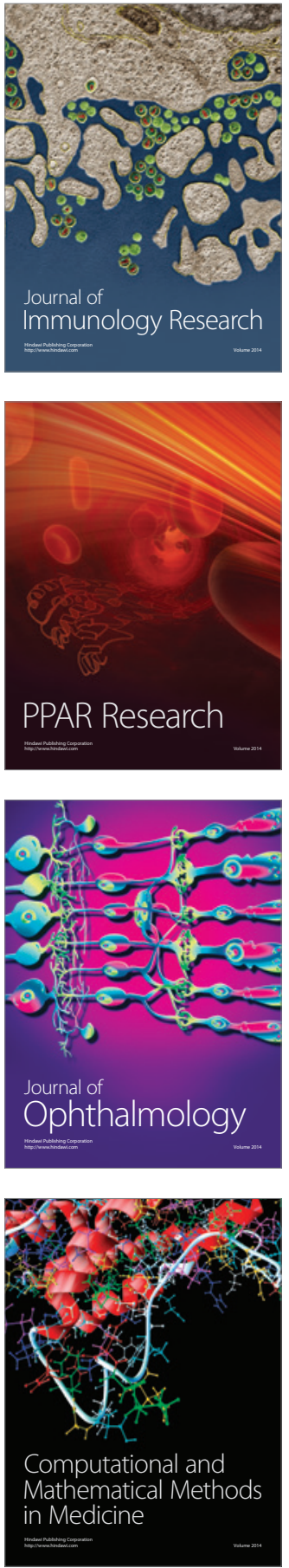

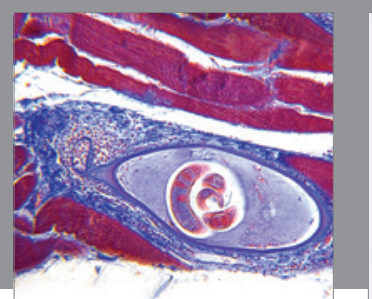

Gastroenterology Research and Practice

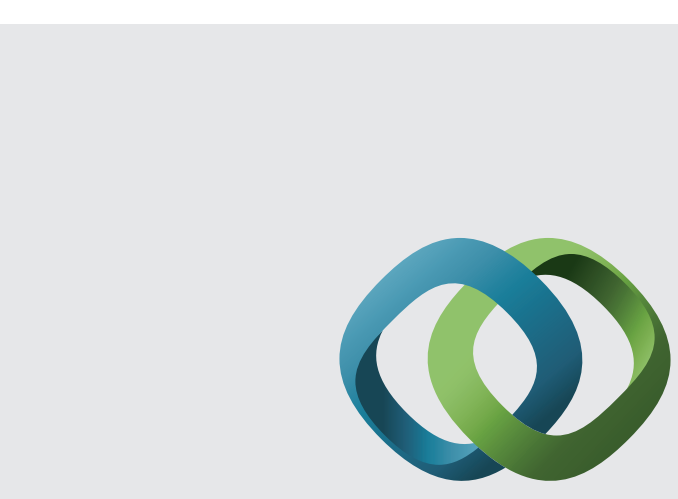

\section{Hindawi}

Submit your manuscripts at

http://www.hindawi.com
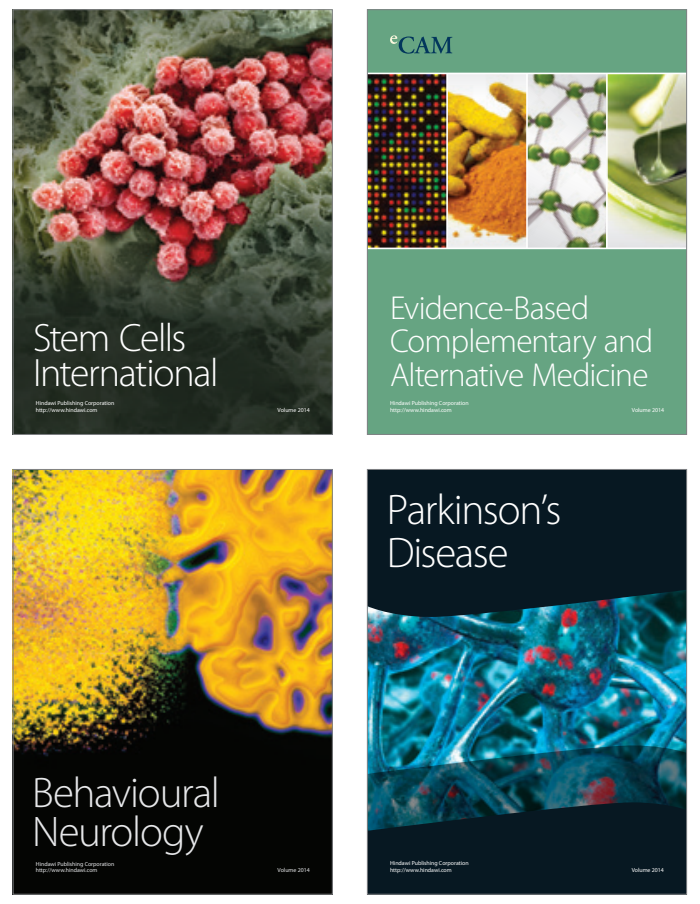
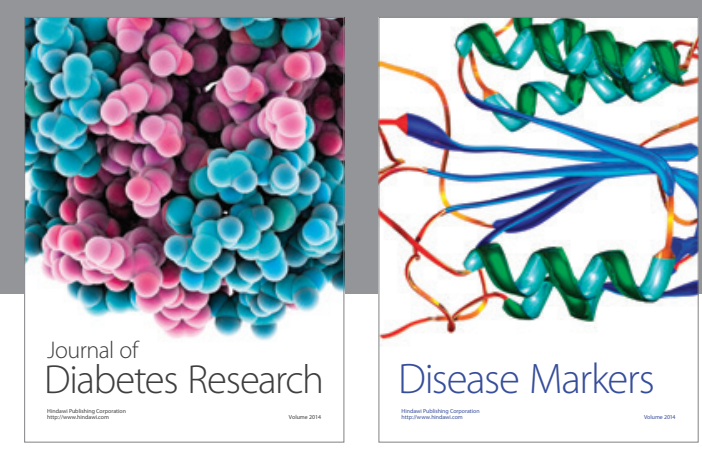

Disease Markers
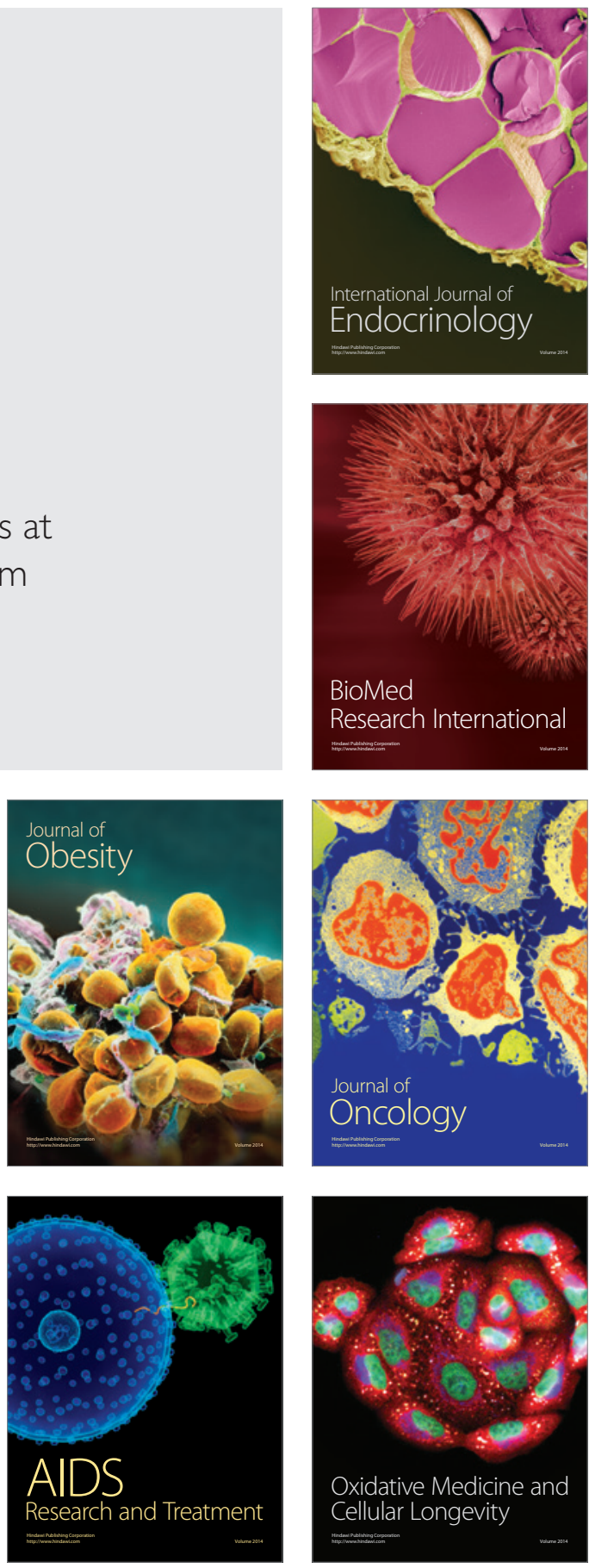